\title{
DISPOSITION MÉTHODIQUE
}

DES

\section{DIATOMÉES D'AUVERGNE}

PAR

\section{Le Frère HÉRIBAUd JOSEPH}

YROFEASEUR AU PRNSIONYAT DE CLERMONT-FERRAND

LAURÉAT DE L'INSTITUT DE FRANCE

(Acadèmie des Sciences)

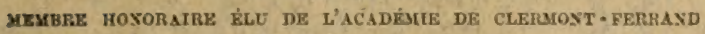

ET DE LA SOCIḰTÉ BOTANIQQUE DE FHAXCE

DEO scientiarum Dsmino

laus et gloria.
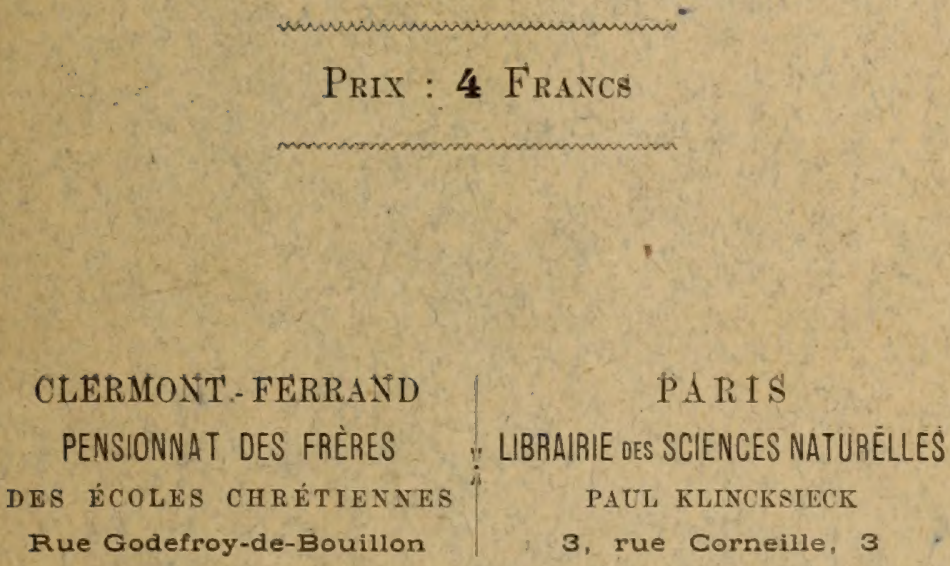

DISPOSITION MÉTHODIQUE

DES

\section{DIATOMEES D'AUVERGNE}





\section{DISPOSITION MÉTHODIQUE}

DES

\section{DIATOMÉES D'AUVERGNE}

$$
\text { PAR }
$$

\section{Le Frère HÉRIBAUD JOSEPH}

YROPEASEUR AC PENSIONNAT DE CLERMONT-FERRAND

LAURÉAT DE L'TNSTITUT DE FRANCE

(Acadèmie des Sciences)

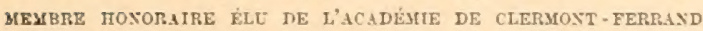

ET DE LA SOCIÉTE BOTAxtQ̨e DE FRAsCE

DEO scientiarum Domino

laus et gloria.

\section{PrIX : 4 Francs}

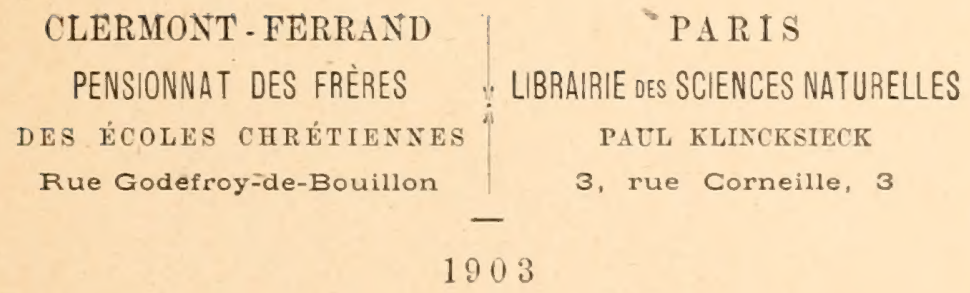





\section{LIERAEV \\ NEW YORE \\ PRÉFA CE}

Après avoir consacré une quinzaine d'années aux Diatomées vivantes et fossiles de l'Auvergne, nous avons pensé qu'il y aurait quelque in térêt à publier le tableau récapitulatif des faits acquis au cours de nos labeurs.

D'autre part, nos dépôts diatomifères, que nous avons étudiés avec le plus grand soin, nous ayant donné une très belle série de formes inédites, et un assez grand nombre d'espèces non inentionnées dans notre Mémoire de 1893, il nous a paru utile de grouper l'ensemble des résultats, en les disposant sous une forme méthodique.

Réunir et classer les faits épars dans nos modestes publications diatomiques, tel est done l'objet des pages que nous offrons en toute confiance aux diatomistes.

La classification suivie dans ce catalogue, ne diffère pas sensiblement de celle des Diatomées 
d'Auvergne (1893). Les quelques modifications introduites nous ont été suggérées par les travaux récents de MM. Clève, Van-Heurck et des commandants Peragallo.

Pour justifier le titre de cette publication, nous avons classé les espèces et les groupes plus ou moins élevés, d'après leurs affinités naturelles, seule disposition vraiment rationnelle.

Nous prions notre savant ami, M. le Commandant Maurice Peragallo, d'agréer nos meilleurs remerciements, pour nous avoir prêté le concours de son profond savoir, dans l'arrangement méthodique de nos petites Algues.

Pensionnat de Clermont-Ferrand, 25 Jirs 1903.

F. Héribaud Joseph. 


\section{DIATOMÉES D'AUVERGNE}

\section{Famille unique. - DIATOMÉES}

Algues colorées très petites, isclées ou réunies en nombre plus ou moins grand par des attaches gélatineuses, formant alors des thalles visibles et simulant des Algues supérieures.

Endochrôme composé de chlorophylle et d'une substance particulière brune nommée diatomine.

Frustule normal constitué par une seule cellule, dont la membrane est siliceuse, composée de deux valves rigides, l'une un peu plus grande que l'autre, réunies par un anneau connectif capable de se développer.

Membrane cellulaire entourée d'une enveloppe gélatineuse (coléoderme), servant quelquefois à réunir les frustules en rubans (Fragilaria) ou en tubes (Melosira); le plus souvent, les prolongements du coléoderme aident à fixer les frustules aux corps immergés.

L'intérieur de la cellule contient, outre l'endochrôme, un liquide incolore (de l'eau probablement), un nucléus et des gouttelettes huileuses. L'endochrôme est disposé en plaques plus ou moins perforées, ou en petits gra. nules arrondis.

Multiplication par division géminipare: l'anneau connectif s'élargit, le noyau se dédouble et deux nou- 
velles valves se forment jointives, autant que leur surface le permet, et opposées aux deux valves primitives.

Reproduction par conjugaison, de différentes manières suivant les espèces. Ce mode de reproduction est encore assez obscur et exige de nouvelles observations.

A l'état vivant, les Diatomées habitent les eaux douces et salées, les eaux thermales dont la température est comprise entre 0 et $45^{\circ}$ centigrades. A l'état mort, on les trouve dans les vases des fossés, des mares, des étangs et des lacs, dans le sol des prairies turbeuses, ainsi qu'à l'état fossile.

Pour tous les détails concernant la nature, la récolte et l'étude de ces microphytes, voir l'Introduction aux Diatomées d'Auvergne, pages 7-40.

La famille des Diatomées se divise, d'après les caractères de l'enveloppe siliceuse, en trois sous familles : les Raphidées, les Pseudo-raphidées et les CryptoRAPHIDÉES.

\section{Sous-Famille I. - RAPHIDÉES}

Frustule présentant, au moins sur une des valves, un vrai raphé, ou ligne brillante sur le fond lisse de la valve, séparé en deux parties par un nodule central et deux nodules terminaux. Le raphé peut être double.

\section{$1^{\text {re }}$ Tribu, - ACHNANTHÉES}

Frustule à valves dissemblables, une seule portant un raphé et des nodules, l'autre un espace hyalin dépourvu de sculpture (pseudo-raphé), linéaire ou plus ou moins élargi, central ou excentrique. 


\section{Genre Rhoicosphenia Grixom 1860:}

Frustule cunéitorme et genonillé; valves cuncifurmes et courbées parallilement an petit axe: face connective également cunéiforme, et montrant des cloisons parallèles aux valies. (Eaux douces et saumâtres).

Rh. curvata Grun. | Rh. Tan-Heurckii Grun.

\section{Gexie Achnanthes Bory de ST-Theest 1822}

Frustule courbé parallèlement au petit axe des valves, non cloisonué; ralres sýmétriques, courbées parallèlement au petit axe; raphé de la valve inférieure droit on légèrement ondulé, placé suivant l'axe de ș̣métrie de la valve; pseudo-raphé quelquetois excentrique. (Eaux douces et saumâtres).

a) Valve inférieure stauronéiforme.

Ach. coarctata Grun.

- subsessilis Ktz.

Ach. hungarica Grun.

- exigua Grun.

b) Frustule présentant sur un des côtés une cloison semi-circulaire; stries de la valve supérieure interrompues sur l'emplacement délimité par la cloison.

Ach. Flahaulti F. Hérib.

- lanceolata Grun.

- - var. elliptica $\mathrm{Cl}$.

Ach. Peragalli F. Hérib. et Br.

- joursacense F. Hérib.

c) Frustule sans cloison; valve inférieure stauronéiforme.

Ach. trinodis Grun.

- gibberula Clère.

- exilis Ktz.

- microcephala Grun.
Ach. minutissima Ktz.

- Biaso'ettiana Grun.

- delicatula Grus. 


\section{Gexre Achnanthidium Grexow (1880)}

Frustule tordu et courlć parallelement au petit axe; raphé et psemio-raphé sigmoides, et aboutissant sur les bords de la valve et non aux extrémités. (Eaux douces).

Ach, flesellum Bréb.

Ach. Alexellum var. alpestris $\mathrm{Br}$.

\section{Genre Gocconeis Ehrenberg (1835)}

Frustule courbé parallèlement au grand axe des valves; valves elliptiques ou subquadrangulaires; raphé et pseudo-raphé droits ou sigmoïdes, passant par le centre des valves. (Eaux douces et salées) ${ }^{(1)}$.

a) Frustule sans anneau marginal interue.

Coc. molesta Ktz.

b) Frustule muni d'un anneau marginal interne et crénelé.

Coc. tenuissima Næxg.

— trilineata F. Hérib.et M.P.

- californica Grun.

- salina Rab.

- Pediculus Ehrb.

- - var. rotunda nob.

- Bonnieri F. Hérib.

- Placentula Ehrb.

- var. minor nob.

Coc. lineata Grun.

- - var. rotunda nob.

- - var. englepta Grun.

- - forma minor nob.

- intermedia F. Hérib.et M.P.

- - var. minor nob.

- Rousii F. Hérib. et Br.

- - var. minor nob.

- speciosa Greg.

(1) On devrait peut-être séparer les espèces qui ont le raphé sigmoïde pour en faire un nouveau genre, comme on séfare les Achnanthidium des Achnanthes et les Pleurosigma des Navicula. 


\section{$2^{\circ}$ Tribu, - NAVICULÉES}

Frustule à valves semblables, portant chacune un raphé ordinairement droit, plus rarement ondulé ou sigmoïde, également développé des deux côtés du nodule médian, dans le sens de la longueur de la valve.

\section{Section Iro - NAVICULÉES (rraies)}

Frustule ni tordu ni caréné; valves droites, non sigmoïdes.

\section{Genre Mastogloia Thwaites (1848)}

Frustule possédant deux diaphragmes, composés chacun d'un anneau marginal divisé en cellules; valves rhomboidales ou allongées; cellules du diaphragme inćgales, formant un rang de chaque côté de la valve, celles des extrémités, de forme triangulaire. (Eaux douces et salées).

Mast. Dansei Thw.

Mast. Smithii Thw.

\section{Gexre Navicula Bory de St-Vincext 1822}

Raphé droit ou légèrement ondulé, formé quelquefois de deux lignes, dont l'une beaucoup plus fine que l'autre, non compris entre des bourrelets siliceux notables; nodule central petit, relativement it la longueur de la valve, non dilaté en stauros allant jusqu'aux bords de la valve; valves ne présentant pas de plis ou sillons profonds, mais simplement des interruptions de la striation ou de légers sillons; striation non régulièrement décussée.

\section{Grodpe des MiCrostigMaticées}

Frustule très petit, ou à valves striées finement. 
1. Mrnutisstme. - Espèces trop petites pour que leurs caracteres peu risibles, même it un fort grossissement, puissent permettre de les classer dans les autres catégories. (Eaux donces et salées).

Nav pelliculosa Hilse.

- atomus Grun.

-- minuscula Grun.

-. atomoides Grun.

- falaisensis Grun.

- minima Grun.

- seminulum Grun.

- - rar. fragilarioides Grun.
Nar. Rotæana Grun.

- - var. oblongella Grun.

- - var. minor Grun.

- binodis T. Sm.

- perpusilla Grun.

- trinodis IV. Sm.

- Creguti F. Hérib.

- - var. lanceolata nob.

2. Fustarorm. - Valves étroites, lancéoléeslinéaires, très finement striées. (Eaux douces et salées). Nav. gibbula Clève.

- - rar. cantalica nob. Nav. crassirostris Cl. et Grun.

3. BAcille. - Valves bacillaires, à striation fine; nodules terminaux renforcés. (Eaux douces).

Nav. Pupula Ktz.

- - var. minuta Ktz.

- - var. major nob.

- lævissima Ktz.

- - var. elongita nob.

- lepida Greg.

- bacilliformis Grun.
Nav, Bacillum Ehrb.

- - var. minor V. Heurck.

- pseudo-bacillum Grin.

- - var. major nob.

- americana Ehrb. (1)

- - var. bacillaris nob.

— - forma minor nob.

4. Limos.e. - Diffèrent des Bacillce par leur contour plus ou moins ondulé, et par un léger sillon soltvent marginal et peu visible. (Eaux douces).

(1) Le Navicula americana est souvent consiléré comme formant une section à part, celle des AMÉricaNéEs. 
Nav, rentricosa Donk.

- - rar. cuneata nob.

- - rar. decrescens nob.

- - forma minor V. Heurck.

- Heribandi M. Per.

- limosi Kitr.
Nav. limosa ra:, gibberula Grun.

- - var. subinflata Grun.

- - var. undulata Grun.

- - var. curta Grun.

- - forma major nob.

5. Formos a: - Valves plus on moins renflées, rhomboüdales, filiptiques, rostrées on capritées; sillons latéraux fins mais visibles. - Les espèces de ce groupe sont comprises par Clive lans le genre Culoncis. Eaux douces et salées).

Nav. patula W. Sm.

I Nav. amphisbæna Bory.

6. Affin. E. - Stries fines, parallèles, interrompues par un sillon submarginal assez large, farallide aux bords de la valve, et accompagné de plusieurs autres de plus en plus faibles à mesure qu'ils se rapprochent des bords. - Ce groupe forme le genre Neidium Clère et Pfitz. (Eaux douces et saumâtres).

Sav. producta $\mathbb{T}$. Sm.

- amphirhynchus Ehrb.

- - var. major $n$ rob.

- affinis Elirb.

- - rar. undulata Grun.

- amplista Ehrlo.

- - var. minor nob.

- firma Ktz.

- Iridis Ehrb.

- var. angistata nob.

- - var. subproducta nob.
Nar. Columnaris Ehrb.

- Hitchcockii Ehrb.

- amphigomphus Ehrb.

- bisulcata Lag.

- - var. major nob.

- dubia Ehhrb.

- Peisonis Grun.

- dilatata Elrrb.

- dubitata F. Hérib. et M. P.

7. Crassinervide. - Valves lancéolées, à striation fine, uniforme, presque parallèle au petit axe, moins distinctement striées parallèlement au grand axe; présentant sourent la forme craticulaire. (Eaux douces et saumâtres). 
L.es Crassinerice pourraient être réunies an Taricula Jifinsoniana Grev. pour reformer le genre Stictodesmis.

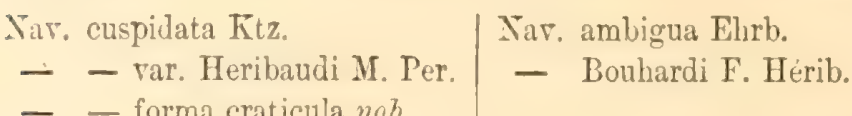

- - forma craticula nob.

8. Seriante. - Valves lancéolées; stries fines, subparallètes, diminuant d'intensité des hords vers le raphé. (Eaux douces).

Nar. serians Ktz.

- - var. Peragalli nob.

- - var. minima Grun.

Nar. serians var. minor Grun.

- exilis Grun.

9. Sculpte. - Valves lancéolées capitées; stries radiantes, décroissant d'intensité jusqu'ì disparaitre, puis reparaissant près du raphé en une double ligne de points très visibles qui accompagne le raphé. (Eaux saumâtres).

Nav. Malinvandi F. Hérib.

- sculpta Ehrb. Nar. sphærophora Rtz.

- - var. minor nob.

10. Stauroneide. - Valves à aspect staurunéiforme; stauros formé, soit par le raccourcissement des stries médianes, soit par leur écartement. Eaux douces et saumâtres).

Nav. Gendrei F. Hérib.

- tuscula Grun.

- nivalis Ehrb.

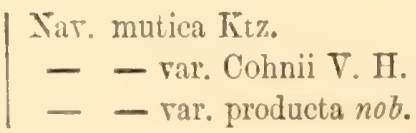

11. Complexe. - Valves non stauronéiformes, souvent engaincées, à zone connective plissće. (Eaux saumâtres et thermo-minérales).

Nar, aponina Ktz. 
Groupe des LINEOLAT.E

Valves lancéolées étroites, à striation un peu forte, ordinairement formée de côtes finoment divisées en travers.

1. Radios.e. - Valves lancéolées, plus ou moins allongées, à extrémités variables, à stries centrales radiantes. - Les Radiose représentent le type des $N^{2} a$ vicula vrais. mâtres.

a) Stries tontes radiantes. (Eaux donces et sanl-

Nar. lanceolata Ktz.

- rostellata Kitz.

- - var. minor V. Heurck.

- slesvicensis Grun.

- gastrum Donk.

- - rar. elliptica nob.

- - rar. major nob.

- Gomontiana F. Hérib.

b) Stries radiantes au centre et droites aux extrémités. (Eaux douces et saumâtres).

X̌ar. Corbieri F. Hérib.

- joursacensis F. Iteril.

- Reinhardtii Grun.

- - rar. elliptica nob.

c) Stries terminales courbées en crochet. Eaux douces).

Tar. Costei F. Hérib.

- - var. bacillaris nob.

d) Stries centrales radiantes, les moyennes droites et les terminales convergentes. (Eaux douces et saumâtres).
Nar. Chaberti F. Hirib.

- Placentula Elırb.

- var. major nob.

- anglica Ralfs.

- dicephala W. Sm.

- - var. minol W. Sm.

- acephala M.P. et F. Hérib.
Nar. triangulifera M. P. F. Hér.

- dichito-lidiatia Grex.

- - val. obesa nob.

- Cyprinus II. Sm. 
A. - Stauronéiformes

Nav. peregrina Heib.

- - var. obtusa nob.

-. - var. fossilis nob.

- gracilis Elırb.

- cincta Kitz.

- Heufleri Grun.

- leptocephala Bréb.

- menisculus A. Sch.

- - var. upsalensis Grun.

- - var. minor nob.
B. - Non stauronéiformes Nav, viridula $\mathrm{K} t z$.

- radiosa Kt\%.

- - rar. acuta Grun.

- tenella Bréb.

- rhsncocephala Ktz.

- Gregaria Donk.

- humilis Donk.

- cryptocephala Ktz.

- pnmila Grun.

- perminuta Grun.

2. Directe. - Valves allongées; stries droites.

Nar. longa Greg.

GrotPE DES COSTAT.E

Valves a côtes plus ou moins robustes, ne portant ni points, ni perles, ni divisions fines.

1. Pinnularie. - Valves de forme généralement hacillaire, ćlargies ou ondulées, munies de côtes lisses ou indistinctement granulées. (Eaux douces).

a) Distantce. - Côtes très fortes et très espacées. Nav. lata Bréb.

- - rar minor nob.

- megaloptera Ehrb.

- costata Ehrb.

Nav. borealis Ktz.

- - var. major nob.

- - var. minor nob.

b) Complexce. - Valves bacillaires; côtes robustes, quelquefois séparées les unes des autres; raphé complexe. (Eaux douces).

Nav. rupestris Ktz.

- viridis Ktz.

- - rar. curta A. Sch.

- var. commutata Grun.
Nav. viridis forma anomala nob.

- nobilis Ehrb.

- - var. gracilis nob.

- gentilis Donk. 
c) Majora. - Valves linćaires, à centre sourent un peu renflé; raphé simple, plus ou moins oblique; aréa ordinairement peu développée. (Eaux douces et saumâtres).

Nar. Dactylus Ehrb.

- gigas Ehrb.

- - rar. gracilis nob.

- major Kitz.

- - var. horridanob.

- - rar. interrupta nob.

- - vir. conteryenlisima nob.

- transrersa A. Sch.

- subacuta Ehrb.

d) Tabellarice. - Talves linéaires, étroites, souvent gibheuses au centre et aux extrémités; aréa modérément large, souvent stauronéiforme. (Eaux douces et saumâtres).

Nar. decurrens Ehrb.

- - rar. hyalina noб.

- - var. curtecostata nob.

- gibba Ehrb.

- - var. hyalina nob.

- tabellaria Ehrb.

- interrmpta W. Sm.

- Lereillei F. Férib.
Nar. Dariana A. Sch.

- - var. miocenica nob.

- aquitania F. Hérib. et Br.

- - rar. undulata nob.

- Porrecta Ehrb.

- Esor Ehrb.

- - var. recta nob.

- Olirieri F. Hérib.

e) Divergento. - Stries du milieu de la valve très difergentes; aréa ordinairement stauronédiome. (Eaux douces).

Nar. notata M. Per. et F. Hérib.

- Brebissonii Ktz.

- - rar. diminnta Grun.

- - var. oralis H. Per.

- - var. subproducta Grun

- - var. elongata nob.

- microstauron Ebrb.

Nar. macra Grun.

- divergens $\mathbb{T}$. Sm.

- - var. prolongata nob.

- - var. unclulata nob.

- - var. undulato-cuneala nob.

- cardinalis Ktz.(1)

- Legumen Ehrb.

- - var. ris-undulata V.H.

(1) Cette belle espice est placće ì tort, par Clive, dans la section des Compleze. 
f) Capitate. - Valves ordinairement stauronéiformes, à extrémités rostrées ou capitées, et à contour plus au moins ondulé. (Eaux douces) ${ }^{(1)}$.

\begin{tabular}{l|l} 
Nar. mesolepta Ehrb. & Nar. bicapitata Lag. \\
- - var. Saintignyii F. Hérib. & - - var. hybrida Grun.
\end{tabular}

- - var. stauroneiformis Gr.

- decrescens F.Hérib. et M.P.

- gracillima Pritch.

- - var. lucida neb.

- Termes Ehrb.

- - var. stauroneiformis V. H.

- nodosa Ktz.

- biceps Greg.

- - var. sulcata nob.

- subcapitata Greg.

- - var.stauroneiformis Gr.

- - var. paucistriata Grun.

- appendiculata Ktz.

- - var. irrorata Grun.

- Brannii Grun.

- - var. moissacensis nob.

- globiceps Greg.

g) Brevistriatce. - Aréa large, quelquefois stauronéiforme; côtes très courtes. (Eaux douces et salımâtres).

Nar. parra Grun.

- acrosphæria Bréb.

- - var. minor nob.

- - var. bacillaris nob.

- - var. elongata nob.

- - rar. badeana nob.

- - var. læris nob.
Nav, brevistriata Grun.

- hemiptera Ktz.

- - var. Bielawskii nob.

- - var. gibba nob.

- - var. angusta nob.

- hybrida MI. Per. et F. Hérib.

- acuminata $\Pi$. Sm.

h) Abbrevice. - Aire centrale large, aire axiale plus étroite; stries rayonnantes, obscurément ponctuées. (Eaux salées et saumâtres).

\begin{tabular}{l|l} 
Nav. cellesensis F. Hérib. & Nar. basalt. rar. undulata nob.
\end{tabular}

- basaltæproxima F. Hérib. - - rar, longistriata nob.

\section{GROUPE DES GRANLLAT.玉}

Stries distinctement granulées; aire axiale peu développée, aires latérales nulles. Eaux douces et salées.

(1) Nous comprenons, duns cette section, celle des Gracillima de Clère. 
Nav. Renauldi F. Hérib.

- - var. major nob.

- pusilla W. Sm.

- arverna M. Per. ct F. Hérib.

- --var. stauroneiformisnob.

- scutelloides Grun.
Nar. scut, var, minor A. Sch.

- amphibola $\mathrm{Cl}$.

- - var. perrieri nob.

- - rar. stanroneiformisnob.

- Berriati.F. Hérib.

- - var. minor nob.

\section{Genre Trachyneis Clève (1894)}

Erustule ordinairement développé sur la face connective; valve bacillaire ou rhomboïdale allongée; raphé souvent sinueux, avec une aire axiale inégalement développée des deux côtés lu raphé; aire centrale stauronéiforme, souvent accompagnée de sillons ou aires latérales sinueuses; stries radiantes, formées de granules allongés et décussés.

Les Trachyneis sont souvent considérés comme groupe des Naviculées sous le nom d'Asprce. (Eaux salées).

Tr. scotica Clére.

Glaxre Stauroneis Enrexberg (1843)

Nodule central dilaté transversalement en stauros; stries ordinairement fines, plus ou moins distinctement granulées.

1. Pleurostauron. - Frustule cloisonné. (Eaux douces et saumâtres).

St. parrula Grun.

— antediluviana M. P. F. Hérib. $\mid \begin{aligned} & \text { St. Legumen Ehrb. } \\ & \text { - acuta W. Sm. }\end{aligned}$

- Smithii Grun.

- jaranica Gr. val. arveruense nob.

2. Stauroneis. - Frustule non cloisonné. (Eaux douces et salées). 
St. Baileyi Ehrb.

- Bruni F. Hérib. et M. Per.

- amphilepta Ehrb.

- platystoma Ehrb.

- mesopachya Ehrb.

- Plianicenteron Elrib.

- var. crassa nob.

- var. lanceolata nob.

- var. gracilis nob.
St. gallica F. Hérib, et MI. Per.

- gracilis W. Sm.

- acntinscula M. Per. F. Hérib.

- anceps Ehrb.

- - var. amphicephala Ktz.

- - var. linearis Grun.

- rar. hyalina nob.

- dilatata W. Sm.

- quadrata F. Hérib. et M. Per.

\section{Genre Diploneis Ehrexberg (1841).}

Valves ovales ou panduriformes; raphé entouré de bourrelets siliceux, séparés de la valve par un sillon profond; nodule central carré, allant jusquaux sillons; côtes plus ou moins robustes, quelquefois ponctuées ou granulées, ou divisées en travers par des sillons parallèles.

1. Elliptica. - Valves non contractées au milieu, pius ou moins ovales; côtes ponctuées ou granulées. (Eaux douces et salées).

Dipl. elliptica Ktz.

- - Var. oblongella Nreg.

- - var. minutissima Grun.

- - var. major nob.
Dipl. elliptica forma minor nob.

- oralis Hilse.

- Pagesi F. Hérib.

- Smithii Bréb.

2. Didymce. - Valres rectilignes allongées, à extrémités arrondies ou plus ou moins panduriformes. (Eaux salées).

Dipl. recta F. Hérib. et Br.

- bomboides A. Sch.

Dipl. homboides var. minor no3.

- - var. media nob.

Gexhe Van-Heurckia de Bribrson 186s

Nodule central petit; raphé entouré de chaque côté 
de nerrures siliceuses; nolules terminaux allongés; stries trè fines, parallites un lénèrement radiantes, formées de points fins et bien éganx, de sorte qu'on aperguit égalem nut une striation longitulinale parallè.e au raphé. (Eaux douces et saumâtres).

r.-H. vulgare V. Heurek.

- - var. lycustris Br.

- lineolata Elrb.

- crassinervia Breb.
V.-H. rhomboides Ehrb.

- Julieni F. Hérib.

- - var. elliptica nob.

\section{Gexre Golletonema de Bréblanos 1849)}

Valve l'́çèrement as rmétrique, ayant un côté un pen plus large que l'autre; nolules terminaux éloignés des extrémités de la valve, et entourés par les stries; frustules engainés. (Eaux douces).

Coll. Cesatii Rab. (1)

\section{Gexre Amphipleura Ketzing (1814)}

Frustule fusiforme; valyes très allongées; raphé à deux petits segments rejetés tout ì fait vers les extrímités; nodules terminaux allongés. (Eaux douces).

Amph. pellucida Ktz.

\section{Sectiox II. - PLEURosigMéES}

Valves ordinairement sirgmoïles. ainsi que le raphé: aire axiale nulle; aire centrale nulle on très petite; striation composée de petits points alignés formant des stries rectangulaires, ou décussés et domnant alors des stries suivant trois directions.

(1) Dans les Dintomées d'A Avergne (1893), et dans notre premier Mémoire sur les Diatomées fossiles d'A uvergne? 1902), les genres Diploneis, Vun-Heurchia et Colletomema, sont compris dans le genre Nuvicula. 


\section{Gexre Pleurosigma W. Smith 1853;}

Valves symétriques par rapport au centre; frustule ni tordu, ni caréné; stries croisées à angle droit perpendiculairement et parallèlement au raphé. Eaux douces et salées.

$\mathrm{Pl}$. attenuatum Ktz.

- acuminatum Grun.

Pl. Spencerii TV. Sm.

- Kützingii Grun.

- - var. scalproides Rab.

\section{SECTION III. - AMPHITROPIDEES}

Valves plus ou moins carénées, à contour non sigmoïde; raphé droit ou sigmoïde, 'central ou latéral, placé sur la carène; stries rayonnantes, formées de points non décussés sur les valves, mais qui le sont quelquefois sur la carène.

\section{Genre Amphoropsis Grexow 1883/}

Valves à raphés opposés, c'est-à-dire placés d'un même côté du frustule; raphé liarqué. (Eaux salées).

Amph. recta (Greg.) Grun。 = Amphiprora recta Greg.

\section{$3^{e}$ Tribu. - CYMBELlíes}

Valves semblables, munies chacune d'un raphé plus ou moins arqué ou biarqué, inéga!ement développées des deux côtés du raphé, la partie du côté convexe du raphé (côté dorsal plus dérelopprée et ordinairement plus fortement striée que la partie concave du raphé (côté ventral). 


\section{Gexre Amphora Ehrenberg (1831)}

Valves inégalement développées des deux côtés du raphé, bombées on anguleuses: striation ordinairement différente des deux côtés du raphé, le côté dorsal plus complitement et plus iortenent strié gue le côté ventral.

1. Amphoroida. - Valres cymbiformes ou linéaires, rôté ventral assez développé; striation rohuste, composce de côtes ou de perles formant une striation rayonnante et des lignes continues on ondulées parallìles au raphé; zone simple. (Eaux douces et salées).

Amph. Borneti F. Hérib.

- Proteus Greg.

- oralis Ktz.

- Pediculus Grun.

- - var. exilis Grun.

- - var. major Grun.
Ampl. Ped. var. minor Grun.

- affinis Ktz.

- gracilis Ehrb.

- libyca Ehrb.

- distincta M. P. F. Herib.

2. Intermedice. - Valves bacillaires, à extrémités aiguës ou obtuses, avec ou sans stauros; côté ventral feu développé; stries fines; raphé fortement iufléchi en arrière. (Eaux douces et salées).

Amph, hyalina Ktz.

3. Cymbelloidec. - Valves cymbiformes, côté ventral plus développé; zone complexe. (Eaux douces et salées!. Amph. veneta Ktz.

- salina IV.Su. Amph. Ergadensis Greg.

- acutiuscula lítz.

- Normanii Rab.

\section{Genre Cymbella Agarde (1830)}

Valves ordinairement plates, à zone connective très 
étroite; raphé excentrique et plus ou moins arqué; stries granulées ou finement divisées en travers. (Eaux douces et légèrement saumâtres).

1. Lunatce. - Valves à bords courbés dans le même sens; raplié assez fortement arqué; nodules récurvés vers le bord dorsal.

Cymb. Harioti F. Hérib.

- radiosi M. Per. F. Hérib.

- cymbiformis Ehrb.

- helvetica Kitz.

- hungarica Pint.

- parvir W. Sm.

- maculata Kiz.

- - forma curta Grin.

- Brericri F. IIćrib.

- Panli J. Per.ct F. Hérib.

- cistula Hompr.

- - var. fusidium nob.

Cymb. Creguti F. Hérib.

- Bouleana F. Hérib.

- stomatophora Grun.

- conifera F. Hérib. et Br.

- Foucaudi F. Hérib.

- tumida Bréb.

- Pagesi F. ILerib.

- lanceniati Ehrb.

- aspera Ehrb.

- gistruides Ktz.

- - var, minor $V . \mathrm{H}$.

2. Tumidoe. - Valres courbées en sens contraires; raphé droit ou légèrement arqué; nodules terminaux placés aux extrémités des valves et peu ou point récurvés.

Cymb. alpina Grun.

- - forma minor nob.

- Charetoni F. Hérib.

- Ehrubargii Greg.

- - var.minor V.H.

- - var. conica nob.

- delect. A. Suh.

- cuspidara Iitz.

- - var. minor nob.

- anglica Lag.

- Laubyi F. Hérib.

- - var. lanceolata nob.

- capitata M. P'er. F. Hérib.

- naviculaforms Ancrs.

- amphicephala Nixg.
Cymb. Hanckii V. H.

- - var. fossilis nob.

- obtusa Greg.

- affinis Ktz.

- turgidu'a Ktz.

- meniscus F. IF́rib. M. P.

- - var. majur nob.

- Rhodesi F. Licrib.

- leptriceras Kitz.

- - forma cinta nob.

- forma minor nob.

- subacgnalis Grun.

- lavis Nirg.

- pusilla (irun.

- microcephala Grun. 


\section{Gexre Encyonema Kutzing $18: 33$}

Todules éloignés des extrémités, placés près de la face dorsale, récurvés vers la face ventrale, et entourés par les stries; raphé rectiligne ou peu arqué. Frustules engaînés. (Eaux douces).

Enc. prostratum Ralfs.

-- Grandi F. Hérib.

- turgidum Grun.

- Girodi F. Hérib.

- intermedium MI.P.E.Hérib.

- cæspitosum Ktz.

- Var. latà V. Heurck.

- - var. Auerswaldii V. H.

- Pediculus Ktz.
Enc. ventrizosum Ktz.

- - rar. excisa nob.

- - var. minuta Hilse.

- norvegicun Grun.

- lunula Grun.

- gracile Rab.

- - var. lunata TI.Sm.

- - forma minor Gru».

\section{4e Tribu, - GOMrhonéuÉES}

Frustule à valves semblables, ayant chacune un raphé et des nodules, asỹmétriques par rapport au petit axe, c'est-à-dire à un plan perpendiculaire au raphé, et passant par le nodule central; la partie la plus courte du frustule (partie supérieure) est ordinairement la plus large et de forme variable; la plus longue (partie inférieure) est presque toujours conique; face connective conique, avec la partie inférieure plus étroite.

\section{Gexre Gomphonema Agardh (1824)}

Frustule non genouillé; stries lisses ou granulées.

1. Stigmaticee. - Valves portant un point unilatéral 


\section{bien visible près du nodule central. (Eaux douces et saumâtres).}

Gomph. cantalicum F. Hérib.

- - rar. costalonga nob.

- - var. lepida nob.

- - forma major nob.

- rigidum M. P. F. Hérib.

- Cyguus Ehrib.

- Tibrio Elinb.

- latestriata F. Hérib.

- affinis Kiz.

- Hebridense Greg.

- intricatum Ktz.

- - Tar. pumila Grun.

- - rar. dichotomarirun.

- clavatum Ehro.

- - forma curta V. H.

- commutatum Grun.

- Mustela Ehrb.

- - var curvata nob.

- - forma minor nob.

-- Brebissoni Ktz.

- elongatum W. Sm.

- - var. minor nob.

- geminatum $\mathrm{Ag}$.

- eriense Grun.

- Lar. actminata nob.

- capitatum Ebrb.

- - var. curta nob.

- constrictum Ehrb.

- - - var. subcapitata Gr.

- - var. clongita nob.

- acuminatum Elarb.

- - var. Irigonocephala Ehrib.

- - var. pusilla Grum.

- - var. coronata Ehrob.

- - var. intermedia Gr.

- - var. gigantea nob.
Gomph, acum, rar clarus V. II.

- - rar. laticeps Grun.

- dichotomnm IV. Sm.

- auritum A. Br.

- subclaratum Grun.

- - Tar. acuminata nob.

- - var. major nob.

- angustatum Grun.

- - var intermedia Grun.

- $\quad$ - rar. subazualis Grun rar. producta Grun. micropus Ktz.

- - var. minor Grun.

- - forma major nob.

- tenellum Ktz.

- montanum Schum.

- - var. pumila Grun.

- biventralis M. P. F.Her.

- subtile Ehrb.

- parrilum Kótz.

- - var. lanceolata Elurb.

- var subcapitata V. II.

- Angur Ehrb.

- - var. Gautieri V. H.

- insigne Greg.

- - var. acuminata nob.

- - forma minor Greg.

- semiapertum Grun.

- - rar.Tergestina Grun.

- sarcophagus Greg.

- Urevistriata F. Hér. M. P. 
2. Astigmaticce. - Valves ne présentant pas de point unilatéral prìs du nodule central. (Laux douces tt saumâtres).

Gomph. excissum M. Per. F. Ièr. Gomph. Gilloti F. Hérib.

- parrum F. Hér. M. Per. - areticun Grun.

- oliraceum Ehrb. $\quad$ - Licmophoraoides I.P.F.H.

- accessum F.Hér. M.Per. - exiguum Ktz.

- Kamtschaticum Grun. _ abbreriatum Ktz.

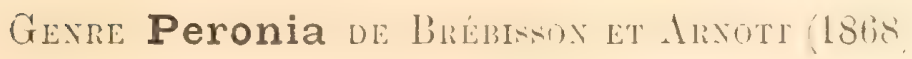

Valyes à nodule central et raphé atrophiés, dont il ne reste que les nodules terminaux et des anorces de raphé, qui parfois manquent à la partie inférieure et même sur toute la valve. (Eaux donces, fossile).

Per. Heribandi M. Per. 


\section{Sors-Fanile II. - PAELDO-RAPHIDÉE'}

Frustule ne portant de raphé sur aucune des valves, présentant quelquefois des uodules très petits accompagnés parfois de sillons ou de côtes simulant un raphé; valves ayant souvent une aréa lisse, linéaire, ou plus ou moins élargie, séprarant les sculptures sur la linne núdiane de la valve, mais ne purtant ni apnendices, ni cornes, ni grosses épines.

\section{- $1^{\text {re Tribu. }- \text { ASTÉRIONELLÉES }}$}

Frustules coniques, très allongés, presque bacillaires, à extrémités plus ou moins renflées, ordinairement réunis en éventail par une des extrémités des ralves; valves striées transversalement, présentant un pseudoraphé étroit, plus ou moins visible, élargi aux extrémités, surtout à celle du contact, qui est presque dépourvue de striation.

\section{Genre Asterionella Hassal (1850)}

Frustules bacillaires, renflés aux extrémités, tant sur la face valvaire que sur la face connective, réunis en

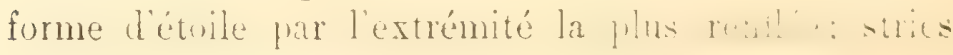
transversales assez fines. (Ealix douces et salées).

Aster. formosa liass. Aster, antiqua F. Hérib. et M. P.

- - var. gracillima Grun. 


\section{$2^{\text {Te THBU }}$ - SINEDREES}

Frustule très allongé, ordinairement bacillaire et symétrique par rapport au plan perpendiculaire au milieu du grand axe; valves striées tranversalement, présentant un pseudo-raphé plus ou moins apparent, quelquefois élargi au milien en pseudo-stauros uni ou bilatéral.

\section{Genre Synedra Ehrenberg (1831)}

Frustules bacillaires, libres, géminés ou réunis en éventail par une des extrémités des valves; face valvaire striée transversilement. prísentant une aréa plus ou moins large, quelquefois stanronéiforme au milieu de la valve de l'un ou des deux côtés.

Les Synedia d'Auvergne ne présentent qu'un seul pseudo-raphé central, et forment le groupe Eusynedrce. (Eaux douces et salées).

Srn. capitata Ehrb.

- joursacensis F. Hér. M. Per.

- Ulna Elrb.

- - var. danica Kítz.

- - var. amphirhynchus Eh.

- - var, bicurrata Grun.

- - var. lanceolata Ktz.

- - var. longissima $\Pi$. Sm.

- - rar. obtusa W. Sm.

- - rar. spathulifera Grun.

- rar. sabaequalis Grun.

- - rar. ritrea Ktz.

- barbatula Ktz.

- Taucherix Kíz.

- - var. truncata Ktz.

- - rar. partula Ktz.

- capitellata Grun.
Syn. delicatissima Wr. Sm.

- - var. mesoleia Grun.

- - rar. angustissima Grun. radians Ktz.

- crotonensis Edw.

- hyperborea Grun.

- acuta Ktz.

- - rar. oxyrhynchus Ktz.

- Acus Grun.

- - var. fossilis Grun.

- - var. ventricosa nob.

- - rar. subtilis Grun.

- rumpens Ktz.

- Ramesi F. Herib.

- pliosenica F. Herib. M. Per.

- affinis Ktz.

- gracilis Ktz.

- closterioides Grun. 


\section{Genre Fragilaria Lyngbye (1819)}

Frustules analogues à ceux des Synedra, mais en général moins longs, réunis en rubans par leurs faces valvaires; face connective exactement bacillaire.

1. Staurosira. - Pseudo-stauros très visible, quelquefois lancéolé; striation asscz forte. (Eaux douces).

Fragil. brevistriata Grun .

- - var. pusilla Grun.

- - var. Mormorum Grun.

- - var. elliptica nob.

- - var. subcapitata Grun.

- lapponica Grun.

- mognalis Lag.

- - var. capitata nob.

- Harrisninii Grun.

- - var. major nob.

- mutabilis Grun.

- Gustarrei F. Hérib.

- binodis Ehrb.

- var. obliqua nob.

- parasitica Grun.

- - var. subconstricta Gr.

- bigibba M. Per. F. Hérib.

- capucina Desm.

- - var. acuminata Grun.

- Crar. acuta Grun.

- - rar. mesolepta Rab.

2. Eufragitaria. - Pseudo-stauros très étroit et peu visible; valves striées en général finement. (Eaux douces et salées).

Fragil, undata W. Sm.

- virescens lialfs.

- var. exigna nob.

- - var. ventricosi nob.

- - rar. elongata nob.

- striatula Livgb.
Fragil. construens Grun.

- - var. capitata nob.

- - Tar. genuina Grun.

- - Tar. pumila Grun.

- - var. Venter Grun.

- - rar. circulare nob.

- elliptica Schum.

- - forma minor Grun.

- minntissima Grun.

- Lancettula Schum.

- intermedia Grun.

- Zeilleri F. Herib.

- - var. nitzschioides nob.

- - forma anomala nob.

- bidens Heib.

- - forma major Heib.

- - forma minor Ileib.

- producta Grun. 


\section{Gexre Ceratoneis Eirranberg $(1840)$}

Frustules de syne Tra plićs sur une des faces connectives, parallèlement à l'axe normal aux plans des valves, et passant par le centre de celles-ci; face connective à flancs parallèles. (Eaux douces).

Cerat. dreus Kíz. Cerat Alcus rar. amphioxys Rab.

\section{$3^{\text {e Tribu. - ECNOTIÉES }}$}

Frustule arqué; face connective rectangulaire; face ralvaire arquée, striće transversalement, présentant des pseudo-nodules marginanx, et quelquefois un pseudoraphé ou des sillons plus ou moins marginaux.

\section{Gexis Actinella Lewris (1865)}

Valves à extrémités inégales, dont l'une au moins est plus large que le milieu de la valve, présentant des pseudo-nodules marginaux et un sillon excentrique rapproché du bord concare. (Enıx douces et saumâtres).

Act. pliocenica F. Hérib. M. Per. | Act. plioc. rar. serpentina nob.

- - var. tenuistriata nob.

\section{Gexre Eunotia Ehrexberg (1837)}

Valves ì extrémités égales; psendo-nodules tout ì fait marginaux; sillon marginal, ordinairement invisible. (Eaux donces et légèrement saumâtres).

1. Eucunotia. - Frustules lihres, parasites, isolés ou réunis par deux; face connective étroite. 
Eun. flexuosa Ktz.

- rar, bicapitata Grun.

- parallela Ehrb.

- lunaris Giun.

- - var. subarcuata Grun.

- - var. excisa Grun.

- - var. bilunaris Grun.

- paludosa Grun.

- polydentula Ehrib.

- - var. fossilis nob.

- - var. bidentula W. Sm.

- - var. tridentula Ehrb.

- Rabenlrorstii Cl. et Grun.
Eun. robusta Ralfs.

- - val. endecaodon Ralfs.

- - var. tetraodon Elirb.

- prierupta Ehrb.

- - var. bigibloa Ktz.

- - var. inflata Grun.

- forma curta Grun.

- monodon Elirb.

- - rar. diodon Elnb.

- impressa Elurb.

- - var. angusta Grun.

- polrglyphis Grun.

- incisa Greg.

2. Himantidium. - Frustules réunis en bandes plus ou moins longues; face connective développée.

Eun. minor Rab.

- pectinalis Rab.

- - var. stricta Rab.

- var. ventricosa Grun.

- var. undulata Ralfs.

- - var. elongara Rab.

- major Rab.

- - var. bidens W. Sm.
Eun. gracilis Rab.

- - var. major nob.

- - var. capitata nob.

- Arcus Ehrb.

- car. plicata nob.

- - rar. hybrida Grun.

- - rar. bidens Grun.

- Faba Ehrb.

\section{$4^{e}$ Tribu. - EPITHÉMIÉES}

Frustules arqués dans la vue valvaire, plus ou moins turgides dans la vue connective; valves ordinairement bombées ou angulaires, couvertes de côtes radiantes étroites mais fortes, et de stries intercostales plus ou moins granulces.

\section{Genre Epithemia ne Brébiscox (18.3}

Valres présentaut un pseudo-raplić assez large et 
visible, formé de deux lignes courbes tangentes au borl ventral, et se rejoignant, sous un angle plus on moins aigu, au milieu de la valre; stries granulces, ou lignes de granules très visibles intercalées entre des côtes fines mais fortes.

Le genre Epithemia so relie au genre Denticula par l'Epithemia ocellata, et au genre IIantzschiu par l'Epithemia turgida. (Eaux douces et saumâtres).

Epith. ocellata Ehrb.

- Argus Ktz.

- - var. alpestris W. Sm.

- - var. amphicephala Gr.

- Sorex Ktz.

- Zebra Ktz.

- - rar. minor nob.

- - rar. proboscidea Grun.

- - var. longicornis nob.

- - var. longissima nob.
Epith. Zebra var. undulata nob.

- turgida Ktz.

- - var. Vertagus Ktz.

- - rar. porcellus nob.

- - var. granulata Grin.

- - rar. crassa nob.

- Westermannii Ktz.

- Hyndmannii W.Sm.

- - var. curta nob.

- - var perlonga nob.

\section{Genre Rhopalodia O. Muller (1895)}

Valres présentant une côte formant arête, et portant au milieu un nodule plus ou moins apparent, et aux extrémités des nodules terminaux moins visibles; striation plus serrée et plus délicate que dans les Eprithemiu. (Eaux douces et saumâtres).

Rhop. constricta W. Sm. (1)

- succincta Bréb.

- gibberula Ehrb.

- - var. producta Girun.
Rh.op. rupestris W. Sm.

- gibba Ehrb.

- - rar. rentricosa Grun.

- - var. parallela Grun.

- - forma lungissima nob.

(1) Les Rhopalodia sont compris, dans nos Diatomées đAurergne (1893), dans le genre Epithemia. 


\section{$5^{e}$ Tribu. - NiTzSCHIFES}

Frustule en général allongé, asymétrique, caréné; valves angulaires, la carène formant l'arête de l'angle; les deux plans de la valve sont réunis près de la carène par des amorces de cloisons plus ou moins longues; vues par les côtés de la valve ces amorces présentent l'aspect de points carénaux arrondis ou allongés; valses striées transversalement.

\section{Genre Hantzschia Grunow (1877)}

Frustule arqué dans la face valvaire, rectangulaire dans la face conuective; carènes marginales, placées sur l'aplomb du bord ventral, et opposées par rapport au plan connéctif, c'est-à-dire du même côté du frustule; pointś carénaux allongés ou prolongés en côtes transversales; nodule médian parfois visible. (Eaux douces et salées).

Hantz. amphioxys Gran.

\begin{tabular}{l|c} 
- - var. intermedia Grun. & - \\
- & - var. virax Grun.
\end{tabular}

\section{Genre Bacillaria Gmelin (1788)}

Carène centrale, c'est-à-dire placée au milieu de la valve, dont les deux faces latérales sont alors égales; valves longuement lancéolées, striées finement en travers; frustules réunis en rubans par la face valvaire. (Eaux douces et salées).

Nous avons repris l'ancien genre de Gmelin, parce que, par la carène centrale, il établit une transition très naturelle entre les Hantzschia et les Nitzschia.

Bacil. socialis Greg.

| Bacil, socialis rar, basaltica nob. 


\section{Genre Nitzschia Hassal $(1845)$}

Carènes excentriques sur les deux valves et diagonalement opposées dans le frustule.

\section{GROUPE DES SCALARIFOR.M.E}

Frustules allongés, droits, fusiformes ou légèrement sigmoïdes; carène presque centrale; points carénaux allongés, et prolongés tous ou en partie sur la valve; ralves lancéolées délicatement strićes en trivrers. Faux douces et saumâtres).

Nitz. dissipata Grun. I Nitz. dissip. var. media Grun.

\section{GROUPE DES SIGMIATE}

Frustules sigmoïdes dans les deux sens (valvaire et connective); carène plus ou moins excentrique; points carénaux ronds et non prolongés sur les valves.

1. Sigmoidce - Carène un peu excentrique; valves sinueuses, les extrémités récurvées d'un même côté; face connective sigmoïde; points carénaux légèrement allongés transversalement. (Eaux douces).

Nitz. sigmoidea Nitz.

Nitz. Brebissonii W. Sm.

_ - var. armoricana Grun.

2. Nitzschiellce. - Carène très excentrique; frustule turgide au centre et à extrémités prolongées, fines, linéaires, courbées du même côté ou en sens différent. (Eaux douces et salées).

Nitz. acicularis W. Sm. | Nitz, ignimontana F. Hérí.

3. Oltusce. - Frustule légèrement sigmoìde; valves 
bacillaires à extrémités atténuées et tournées en sens contraire; carène i inflexion centrale, présentant au milieu de l'inflexion deux points carénaux plus distants que les autres; quelquefois un rudiment de nodule existe entre les deux points. (Eaux saumâtres).

Nitz. obtusa W. Sm.

I Nitz. obtusa rar. scapelliformis Gr.

\section{GrodPE DES LINEARIE}

Frustule bacillaire, à carène très excentrique; face connective bacillaire ou très longuement lancéolée; face valvaire lancéolée on bacillaire, à extrémités plus ou moins acuminées; points carénaux ronds ou à peine allongés.

1. Lanceolatce. - Valves lancéolées, ou lancéoléeslinéaires, plus rarement ovales; carène très excentrique, à points carénaux ronds. (Eaux douces et salées).

Nitz. ovalis Arn.

- inconspicua Grun.

- microcephale Grun.

- fonticola Grun.

- tubicola Grun.

- subtilis Grun.

- fossilis Grun.

- minuta Bleisch.

-- acutiuscula Grun.

- communis Rab.

- - var. obtusa Grun.
Nitz. Frustulum Grun.

- - rar. minutula Rab.

- - var. perpusilla Rab.

- - var. Bulnheimiana Rab.

- Palea Ktz.

- - var. exilis Grun.

- - var. tenuirostris Girun.

- Hantzschiana Rab.

- amphibia Grun.

- - var. Franenfeldii Grun.

2. Linearia. - Valves à carène excentrique, longuement lancéolées ou linéaires, ¿̀ points carénaux ronds ou subanguleux, un peu allongés transversalement; face ronnective bacillaire, quelquefois légèrement rétrécie au milieu, à extrémités atténucées et tronquées. Fanx douces ou saumâtres.) 
Nitz. recta Hantz.

- tenuis Grun.

- linearis W. Sm.

- - var. major V. Heurck.
Nitz. vitrea Norm.

- - var. gallica nob.

- Kittlii Grun.

3. Sipectubilo. - Valves grandes, faiblement arquées, à carc̀ne excentrique; points carénaux un peu prolongés sur la valve. (Eaux saumâtres).

Nitz. spectabilis Rab.

\section{GROUPE RES CONSTRICTE}

Frustule rétréci au milieu; face connective bacillaire ou légèrement contractée, à extrémités plus ou moins arronlies; points carénaux sensiblement prolongés sur la valve.

1. Dubice. - Face valvaire légèrement contractée, ainsi que la face connective; celle-ci à extrémités acuminées, quelquefuis rostrées capitées; points carénaux ronds ou légèrement allongés dans le sens trausversal. (Eaux douces et saumâtres).

Nitz. thermalis Auersw.

- commutata Grun.

Nitz. dubia W. Sm.

2. Bilobate. - Faces valvaire et connective plus contractées que dans la section précédente; carène moins excentrique; points caréuaux plus allongés en général; face connective à extrémités acuminées et récurvées vers le connectif. (Eaux saumâtres).

Nitz. bilobata W. Sm. | Nitz, bilobata var, hy̧brida Gr.

GROUPE DES EPITHEMIOID无

Points carćnaux prolongés par des cưtes lien défi- 
nies; carène tout à fait excentrique; côtes traversant toute la valve, tout en dimiuuant insemsiblement de largeur. (Eaux douces).

Nitz, Denticula Grun.

\section{GROURE DES TRYBLIOYELLAE}

Valves présentant un sillon plus ou moins large, lisse, ou une ondulation longitudinale où les stries sont moins accusées; carène marginale; frustule ordinairement contracté.

1. Apiculato. - Valves linéaires, longuement acuminées, présentant un sillon sur lequel les stries manquent ou sont très affaiblies. (Eaux douces et saumâtres).

Nitz. hungarica Grun. | Nitz. acuminata Grun.

2. Panduriformo. - Valves larges et contractées à la partie médiane, présentant un sillon ordinairement net et étroit; stries granulées, formées de points décussés; carène très excentrique. (Eaux salées).

Nitz. panduriformis Greg.

- - var. lucida nob.

Nitz. constricta Greg.

3. Tryblionellce. - Valves ordinairement larges, contractées ou non au milieu, ondulćes dans le sens de la largeur, mais dépourvues de sillon (1). (Eaux donces et salées).

Nitz. angustata W. Sm.

- calida Grun.

Nitz. Victoria Grun.

- Tryblionella Hantz.

(1) Nous avons compris dans les Tryblionella, les Psendo-Tiyblionella qui n'en diffierent que par la forme des points carinaux. 


\section{Gexre Grunowia Rabenhorst (1864)}

Talve carénée comme dans les Nitzschia, à flancs plus ou moins ondulés, à extrémités capitées; côtes fortes et alternes, s'arritant hrusinement au milieu de la face valvaire; stries intercostales fines et souvent peu visibles.

Grun. Tabellaria Rab.

- sinuata Rab.

Grun. moissacensis E. Hérib.

\section{$6^{\ominus}$ Tribu, - SURIRELLÉES}

Frustule à valves symétriques, présentant, de chaque côté de la valve, une carène plus ou moins marginale, mais dont le bord se projette presque toujours très près des bords de la valve, et ne se voit bien que dans la vue connective; striation formée de côtes plus ou moins fines, terminées au bord de la carène par une cupule ou par une perle, et entre lesquelles sont souvent des stries beancoup plus fines:

\section{Gexre Cymatopleura W'. Smiti (1851)}

Frustule à valves ondulées longitudinalement, à carène marginale; points carénaux gros; stries très fines; pseudo-raphé peu distinct. Eaux donces et sammâtres'.

Cym. hibernica W. Sm.

- - var. major nob.

- elliptica IT.Sm.

- - var. subconstricta Gr.

- - var. constricta Grun.
Cym. ellip. rar, rhomboides Gr.

- Martyi F. Hérib.

- Solea Bréb.

- - var. apiculata Pritch. 


\section{Genre Surirella Turpin (1827)}

Frustule navicnlaire ou tordu en spirale; face connective présentant des ailes formées par les carènes des valves; carènes semi-marginales; striation formée de rôtes plus on moins robustes, terminées sur la carène par une cupule.

1. P'irnatre. - Carènes peu saillantes, presque marginales; striation formée de côtes fines et souvent de stries intercostales. (Eaux douces et saumâtres).

Sur, gra silis Grun.

- - var. minor Br.

- - var. constricta nob.

- - var. eximia nob.

- angusta Ktz.

- - var. contorta P. Petit.

- salina W. Sm.

- ovalis Bréb.
Sur. ovata Ktz.

- - var. pinnata W. Sm.

- - var. minuta Bréb.

- crumena Bréb.

- Patella Ehrb.

- Bruni F. Hérib.

- elegans Ehrb.

- norvegica Ehrb.

2. Rolustre. - Carènes saillantes, semi-marginales; côtes robustes, sans stries intercostales visibles dans le baume. (Eaux douces et saumâtres).

Sur. tenera Greg.

- - var. splendidula Greg.

- splendida Ehrb.

- helvetica Br.

- Pagesi F. Hérib.

- biseriata Bréb.

- - var. elliptica P. Petit.

- - var. linearis IV. Sm.
Sur. bis. rar. subacuminata V.H.

- bifrons Ktz.

- turgida W. Sm.

- saxonica Auersw.

- robusta Ehrb.

- striatula Turp.

- var. Gautieri nob.

3. Spiralce. - Frustule tordu en spirale; axes des deux valves paralliles; côtes rohustes et longues. (Eaux donces). 


\section{Gexre Campylodiscus Ehrexibrg (1841)}

Frustule tordu en sens contraires suivant deux axes rectangulaires passant par le centre de figure du frustule, et situés dans les plans de srmétrie des valves; celles-ci ont, par conséquent, leurs axes courbes; l'axe longitudinal, c'est-à-dire celui qui accompagne le pseudo-raphé, est ordinairement le plus long, mais presque toujours les deux axes sont sensiblement égaux, de sorte que les ralves sont orbiculaires ou quadrangulaires à angles fortement arrondis. (Eaux douces et salées).

Camp. Thureti Bréb.

- costatus W. Sm.

Camp. noricus Ehrb.

Gexre Stenopterobia de Brébisson (1865)

Valves très allongées, linéaires acuminées, carène tout à fait marginale; face connective sigmoïde. (Eaux douces).

Stenop. anceps Lewis.

\section{7e Tribu. - RAPHONÉIDÉES}

Valves couvertes de ponctuations ou de grantilations en général grosses et très visibles, disposées en lignes rayonnantes et parallèles au pseudo-raphé, qui est linéaire ou lancéolé el très apparent.

Genre Opephora P. Petit (1888)

Frustule conique, tant en vue connective qu'en vue 
valvaire; valve présentant des côtes robustes, laissant au milieu de la valve un pseudo raphé très net et plus ou moins large. (Eaux douces et salées).

Op. Martyi F. Hérib.

- - var. robusta nob.

- - var. capitata nob.

- pacifica P. Petit.

Op. pa sifica var, trigona nob.

- cautalense F. Hérib. M. Per.

- - var. capitata nob.

\section{Genre Raphoneis Ehrenberg (1844)}

Valves symétriques, lancéolćes, plus ou moins turgides et apiculées, couvertes de granules formant des lignes rayonnantes et parallèles au pseudo-raphé, qui est très visible. (Eaux salées).

Raph. belgica Grun.

- var. elongata Grun. $\quad-\quad$ - var, minor Grun.

\section{Genre Campylosira Grunow (1882)}

Valves dissymétriques par rapport à l'axe longitudinal, cymbifornes, couvertes de ponctuations éparses quelquefois peu visibles; face connective élargie au centre et aux extrémités ${ }^{(1)}$. (Eaux salées).

Camp. Peragalli F. Hérib.

(1) Le Dr Van-Heurck place le genre Campylosirce dans la tribu des Fragilarices, tribu qui n'a ancune raison d'être puisque les Frogilaria ne different presque pas des Synedia; nons devons aussi faire observer que le genre n'est guire mieux placé dans los Raphoacidées; il serait pentêtre préférable de le réunir au Cymáosira, pour en former une tribu particulicre. 


\section{Tribu. - DENTICULÉES}

Frustule normalement dépourru de cloisons; valres présentant des côtes transversales et lesstries intereostales, interrompues par un pseulu-raphé sonvent peu visible.

\section{Gexre Denticula Kutzrng (1844)}

Face valvaire ordinairement lancéolée, présentant des côtes transversales robustes, et des stries intercostales ponctuées, sans psendo-raphé apparent; face connective rectangulaire it angles arrondis, montrant le prolongement des côtes qui s'y recourhent et riennent se terminer contre l'anneau connectif par une perle très brillante. (Eaux douces, salées et thermales).

Dont, elegans Ktz.

- - var, thermalis Ktz.

Dent. tenuis Ktz.

- inflata W. Sm.

- - var. intermedia Grun.

- frigida Ktz.

- - var. meso'epta Grun.

\section{Genre Diatoma de Candolle (1805)}

Frustule comme dans le renenre précédent, mais à valves souvent plus bacillaires, à côtes moins fortes, et qui, sur la face connective, s'amincissent vers l'anneau, au lieu de s'y terminer par un granule brillant; frustule muni quelquefois de cloisons internes courbes, situées entre la valve et le connectif. (Eaux douces).

Diat. pectinale Ktz.

- - var. capitata nob.

- - forma elongatum Ktz.

- rulgare Bory.

- - var. lineare W. Sm.

- Mesodon Ktz.

- hycmale Heib.
Diat. hyemale var. acula nob.

- anceps Grun.

- - var. anomalum W. Sm.

- Ehrenbergii Ktz.

- - rar. gracile IV. Sm.

- elongatum Ag.

- tenue Ag. 


\section{Genre Meridion Afaridh (1824)}

Le genre Mrrition ne diffère du geure Diatoma qu'en ce que le frustule est cunéiforme, tant dans la vue valvaire que dans la vue connective; frustules réunis en bande spiralée. (Eaux douces).

Merid, circulare Ag.

Merid, constrictum Ralfs,

\section{9* Tribu, - TABellariées}

Frustule présentant, dans la face connective, des cloisons ou des fausses cloisons parallèles aux valves.

\section{Genre Tetracyclus Grunow (1862)}

Valves munies de côtes transversales fortes, plus ou moins courbes, et, assez sourent, arec des stries intercostales très fines, interrompues par un pseudo-raphé étroit et peu apparent; fare connective montrant de nombreuses fausses cloisoni., ordinairement alternantes d'un côté à l'autre, et percées d'une ouverture centrale. (Eaux douces et saumâtres).

Tetr. Braunii Grun(1).

- ellipticus (Ehrb.) nob.

- rhombus Ralfs.

- Boryants (Pant.) var. millor nob.

- Lancea (Ehrb.) nob.

- compressus (Ehrb.) $\approx$ iab.

- Lamina (Ehrb.) nob.

- castellatus (Ehrb.) nob.

- - var turris nob.

- tripartitus F. Hérib. et Br.

- - var. gracilis nob.
Tetr. stella (Ehrb.) nob.

- Pagesi F. Hérib.

- stellare F. Hérib. II. Per.

- elegans (Ehrb.) nob.

- - var. esimia nob.

- emarginatus W. Sm.

- - var. crassa nob.

- Peragalli F. Hérib.

- - rar. eximia nob.

- - var. major nob.

- decoratus F. Hérib. et $\mathrm{Br}$.

(1) Le Tetracyclus Braunii Grun. est élevé au rang de genre, par plu. sieurs auteurs, sous le nom de Gomphogramma rupesive A. Br. 


\section{Genre Tabellaria Enrexberg (1839)}

Valves striées transversalement, sans côtes interposćes, à pseudoraphŕ apparent; fausses cloisuns n'arrivant que jusqu'au milieu de la valve. (Eaux douces).

Tab. fenestrata Ktz.

- - var. nodosa Elrb. Tub. floceulosa Ktz.

- - var. trinodis Ehrb.

- - var. biceps Ehrb.

\section{Genre Striatella Agardh (1832)}

Valves munies d'un pseudo-raphé très apparent, dépourvues de côtes; stries très fines; face connective montrant un grand nombre de fauseses cloisons très délicates. (Eaux salées).

Str. Girodi F. Hérib. 


\section{Sols-Famile III. - CRYPTO-RAPHIDÉES}

Frustule à valves circulaires, elliptiques ou angulaires, plus rarement hacillaires, largement arrondies, ne présentant jamais ni raphé, ni pseudo-raphé, ni nodules.

\section{$1^{\text {re }}$ TrIBU, - MÉLOSIRÉES}

Valves circulaires, ne portant ni grosses épines, ni appendices, ni ocelles, quelquefois bombées ou globulaires; face connective rectangulaire, à extrémités plus ou moins arrondies.

\section{Genre Melosira Agardh (1824)}

Valves plates ou bombées, mais non ondulées transversalement; frustules sonvent très développés dans la face connective, ordinairement assemblís en rubans par les faces valvaires.

1. Orthosira. - Face connective étroite; frustules réunis en grand nombre poư former un long ruban solide; valves plates. (Eaux douces et saumâtres).

Melos, undulata Ktz.

- - var, producta A. Sch.

- - var. debilis nob. Melos. Sol Ehro.

- - forma hungarica A. Sch.

- arenaria Moor.

- varennarum N. P. F. Hér.

- Boulayiana M. Per.

- imperfecta F. Hérib.

2. Aulacosira. - Face connective déreloppée; valres 
plates, se joignant par toute la fice connective; irustules formant de longs rubans. (Eaux douces et saumâtres).

Melos. Dickici Kítz.

- minuta Mr. Per. et F. Hér.

- nivalis W. Sm.

- distans Ehrb.

- - var. alpigena Grun.

- tenuissim 8 Grun.

- tenuis Grun.

- lavis Grun.

- crenulata Kitz.

- - var. ambigua Grun.

- - var. Falida Grun.

- var. undulata nob.

- Camusi F. Hérib.

- - rar. conica nos.
IIelos lineolata (irun.

- - var. robusta nob.

- granulata Ehrb.

- - var. australiensis V.II.

- - var. arcuata nob.

- spiralis Ktz.

- - var.hemisphæric nob.

- - var. sphærica nob.

- lyrata Ehrb.

- - Var. lacustris Grun.

- canalifera $\mathrm{F}$. Hérib. et Br.

- var. anastomosans nob.

- striata F. Hérib. et Br.

- Bruni F. Hérib.

3. Lysigonium. - Face connective déreloppée; valves légrement lombies, ì centre plat; frustules réunis en bandes; structure gínirale tris fine. Faux douces et saumâtres).

Melos. orichalcea Mert.

- varians Ag.

- Heribaudi Br.

II elos. Borreri Grev.

- - var. ignimontana nob.

4. Liparogyra. - Valves légèrement bombées, munies d'une couronne de petites ṕpines; stries radiantes ponctuces, ponctuation devenant de plus en plus fine vers le centre, qui est muni de deux à cinq gros granules. (Eaux douces, mousses humides).

Melos. Rieseana Moor.

\section{Genre Cyclotella Kutzing (1833)}

Frustules réunis par deux au plus et jamais en ru- 
ban; face connective ftroite, montrant les ondulations de la face valvaire; valves circulaires, bombées ou ondulées circulairement ou en travers, couvertes de stries rayonnantes, laissant ordinairement un centre lisse ou lécrèrement granulé. (Eaux douces et saumâtres).

Cycl. opereulata Ktz.

- - var. antiqua W. Sm.

- Kutzingiana Chaur.

- - var. gemmatula nob.

- perforata M. Per. F. Hérib.

- Comta Ktz.

- - var. radioza Grun.

- - var. arverna nob.

- Bodanica Eul.

- Meneghiniana Ktz.

- - var. rectangulata Grun.

Cyel. comensis Grun.

- stelligera Cl. et Grun.

- Temperei F. Hérib.

- Charetoni F. Hérib.

- - var. scutiformis nob,

- - rar. radiata nob.

- Iris F. Hérib. et Br.

- - var. oralis nob.

- - var.cocconeiformisnob.

- - var. integra nob.

\section{$2^{e}$ Tribu, - COSCINODISCÉES}

Frustule peu développé en général dans la face connective; valves plates, bombées ou ondulées, couvertes d'une structure de même nature, et semblable sur toute la surface, n'ayant que rarement un centre hyalin et petit.

\section{Genre Stephanodiscus Eirexberg (1845)}

Valves ondulées concentriquement, couvertes de faisceaux de ponctuations fines, séparés par des rayons hyalins, à la uaissance desquels se trouvent de petites épines formant un cercle complet submarginal. (Eaux douces et saumâtres).

Steph. Astræa Ktz. Steph. Hautzschianus Grun. 


\section{Gexre Coscinodiscus Finkiberi (18:38)}

Talres plates ou plus ou moins bombées, ayant la même structure sur tuute la surface des bords au centre, sans rayons hyalins; centre hyalin peu étendu ou nul; hords de la valve portant puelyuetois me couroune d'épines marginales. Faux salin's et thermo-saumâtres, jamais dans les eaux douces).

Cose. radiatus Ehrb.

- exasperans Roth.

- pygmaeus M.Per. F. Hérib. - - rar radiata nob.

- - var. micropunctatanob. - - forma inermis not.

- - var. minutissima nob. $\quad$ - chambonis M. Per. F. Hérib.

\section{$3^{e}$ Tribu. - EUPODISCÉES}

Frustule peu développé dans la face connective; valres circulaires, orales, crénulées, rarement subangulaires, couvertes dime sculpture unilorme, granulie on réticulée, présentant des ocelles plus ou moins marginaux.

Gexre Heribaudia M. Pelinillo (K9:3)

Valres circulaires ì expansions ailées; ocelles nuls, ou, s'ils existent, placés sur la lace connective. (Fossile.

Hérib, ternaria M. Per, (1)

(1) L'Heribaudia ternaria se place à côté dn Cerataulus subangulatus Grov. qui, d'après A. Schmilt, ne peut être consicléré comme un Cerataulus, et devrait appartenir a un autre genre; ces deux especes pourrajent donc être réunies dans le genre Heribaudia. 


\section{$4^{e}$ Tribe. - CH FTHOCÉRÉES}

Frustule hyalin ou à structure excessivement fine; face connective très développée par rapport à la face valvaire; valves circulaires on elliptiques plus ou moins allongées, munies de soies ou dlippines piquantes plus ou moins bifurquées; ralves d'un même frustule souvent dissemblables.

\section{Genre Periptera Eurexberg. (1844)}

Talves dissemblables, elliptiques ou circulaires, l'une d'elles très bombée, lisse ou à bords granulés, l'autre moins bombée et portant une couronne d'épines lamelliformes, plus ou moins bifurquées, placées dans le prolongement de la surface du connectif. (Fossile).

Perip. saxogallica F. Hérib. 


\section{CONCLUSION}

Tos premiires recherches sur les Diatomées vivantrs et fossiles de la flore d'Auvergne datent de 1888. Le nombre des espèces et variétés, relevées dans les publications diatomiques anteriemes it cette date, est de 1:22, comprenant sib liatomés bussiles et itf appartenant à la flore actuelle; les 86 espèces fossiles provienneut des dépôts de Ceyssat, des Rouilhas, de Randanne et de Saint-Saturnin, sommairement étudiés par MII. I'aul Petit et Leudnger-Fortmorel; les 36 Diatomées vivantes ont été trourées par W. Smith, J. Brun, P. Petit et Max. Roux.

Dans notre mémoire de 1893 , nous en avons mentionné 716 , c'est donc une augmentation numérique de 594 espèces ou variétés, parmi lesquelles 126 ont été reconnues nouvelles pour la flore générale.

Eufin, nos deux deniers mémoires sur les Diatomées des dépôts tertiaires du l'ur-de-I)ôme et du ('antal portent le nombre de ces microorganismes de 716 à 908 , comprenant 564 espèces et 344 rariétés bien définies, arec 281 formes inédites, décrites avec soin, et figurées dans douze belles planches dessinées par II. J. Brun, professeur de micrographie à la Faculté de Genève, et par le savant diatomiste français, M. le commandant Maurice Peragallo.

Tel est l'état actuel de la flore diatomique de l'Auvergne. 
Pour aroir l'ensemble des Diatomées du Plateau C'entral, il nous sulfit diajunter à celles de ce catalogue les espreces et les rarietrís des dépôts de la Haute-Loire et de l'Ardèche, qui n'ont pas encore été constatées dans ceux d'Auvergne.

Quant anx l)iatmence rivantes nums ne pensons pas que les recherches ultérieures ajoutent notablement aux esperces déji connues, ì en juger, du moins, jar le résultat insignifiant que nous a donné l'étude récente d'un assez grand nombre le réroltes provenant de statious très diverses.

Toici les Diammées des hépôts tertiaires de la HauteLoire et de l'Ardèche, qui n'ont pas encore été trourées en Aurergne.

Achnanthes ligeriana nov. sp. (dépôt de Ceyssac, Haite-Loire). - lanceolata var. oralis nov. (Le Ranc, Ardèche).

Cocconeis scutellum var. minuissima Grun. (Le Ranc).

Navicula Bacillun var. major nov. (Ceyssac).

- ovata M. Per. (Le Rane).

- halophila var. major nou. (Cerssac).

- radians nou. sp. (Ceyssac).

- heroina A. Sch. (Ceyssac).

- gastroides var, elliptica nov. (Pourchères, Ardèche).

- lambertensis nov. sp. (La Roche-Lambert, Hte-Loire).

Van-Heurckia rhomboides var. amphipleuroides Grun. (La RocheLambert).

Cymbella delicatula Ktz. (Le Rane).

- Ehrenbergii var. conica nox. (Le Monastier, Hte-Loire).

- australiea rar. fossilis nov. (La Rochc-Lambert).

- meniscus var. major noz'. (Le Monastier).

- Bruyanti nov. sp. (La Roche-Lambert).

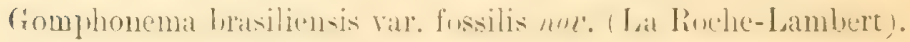

Fragilaria Zeilleri var. densestriata nor. (Ceyssac).

- orata M Per. (Le Rane).

- brevistriata var. capitata noc. (Ceyssac).

- bigibba nov. sp. (Cerssac).

- arualis var. capitata nov. (Le Monastier). 
Ceratoneis antiqua nov. sp. (La Roche-Lambert).

Eunotia polyglyphis var. excisa nov. (La Roche-Lambert).

Epithemia fenestrata M. Per. (Le Ranc).

Opephora Martyi var. minor nov. (Gourgouras, Ardèche).

MIelosira bellicosa nou. sp. (Ceyssac).

- sphærica nov. sp. (Ceyssac).

Cyclotella comta var. decrescens nov. (Ceyssac).

- - var. trinotata nov. (Ceyssac).

- - var. quadrinotata nov. (Ia Roche-Lambert).

- - var. quinquenotata nov. (La Roche-Lambert).

- antiqua rar. mirifica nov. (La Roche-Lambert).

- Guignardi nov.sp. (La Roche-Lambert).

- Temperei var. inermis nov. (La Roche-Lambert).

Terpsinoe trifoliata Clève (Ceyssac et La Roche-Lambert).

Coscinodiscus Butlei H. Peragallo. (Le Monastier et (iourgouras).

Les Diatomées ohservées dans les dépôts de Charay (Ardèche) et de Vals (Haute-Loire) appartiennent à ceux du Cantal.

Le nombre des espèces et varićtés de la liste précédente est de 37 , ce qui donue un total de 945 Diatomées pour le Plateau Central. 



\section{TABLE ALPHABÉTIQUE DES GENRES}

Achnanthes Bory ........ 3

Hantzschia Grun ........, 28

Achnanthidium Grun ...... 4

Actinella Lewis .......... 25

Heribaudia M. Per......... 43

Amphipleura $\mathrm{K} t z \ldots \ldots \ldots \ldots .15$

Mastogloia Thw ......... 5

Amphora ............. 17

Melosira $\mathrm{Ag}, \ldots \ldots \ldots \ldots \ldots \quad 40$

Meridion Ag........... 38

Amphoropsis Grun ..... ... 16

Asterionella Hassal ....... 22

Bacillaria Gmelin......... 28

Campylodiscus Ehrb....... 35

Campylosira Grun .... . . . 36

Ceratoneis Ehrb.......... 25

Cocconeis Ehrb ......... 4

Colletonema de Bréb. ...... 15

Coscinodiscus Ehrb......... 43

Cyclotella Kitz........... 41

Cymatopleura W. Sm...... 33

Cymbella Ag............ 17

Denticula Ktz ........... 37

Diatoma de Candolle....... 37

Diploneis Ehrb........... 14

Encyonema Ktz. ......... 19

Epithemia de Bréb........ 26

Eunotia Ehrb........... 25

Fragilaria Lyngb. . . . . . . . 24

Gomphonema Ag......... 19

Navicula Bory ......... 5

Nitzschia Hassal .... ..... 29

Opephora P. Petit ........ 35

Periptera Ehrb........... 44

Peronia de Bréb. et Arn.... 21

Pleurosigma IV. Sm....... 16

Raphoneis Eurb ......... 36

Rhoicosphenia Grun ....... 3

Rhopalodia O. Mull. ....... 27

Stauroneis Ehrb......... 13

Stenopterobia de Bréb...... 35

Stephanodiscus Ehrb. ..... 43

Striatella $\mathrm{Ag} . \ldots \ldots \ldots \ldots \ldots$

Surirella Turpin .......... 34

Synedra Ehrb ........... 23

Tabellaria Ehrb........... 39

Trachyneis Clève......... 13

Van-Heurckia de Bréb. ..... 14

Conchusion............ 45

Grunowia Rab............ 33 
CLERMONT-FERRAND

IMPR IMERIE

MALLEVAT,

3, PLACE DE LA TREILle 


\section{AUTRES PUBLICATIONS BOTANIQUES}

\section{DU F. HERIBAUD JOSEPH}

Le Puy-de-Dôme et le Cantal ou Tableau comparatif de la Flore des deux départements (1876).

Florure des terrains arrosés par les eaux minérales de l'Au. vergne (1878).

Note sur quelques Menthes observées dans le département du Cantal (1878).

La Flore d'Auvergne (1883), en collaboration-avec mon premier maître en botanique, le très regretté $\mathrm{F}$; Gustave.

Les plantes parasites de la Flore d'Auver rne (1889).

Eléments de Botanique, comprenant l'organographie, la physiologie et les principales familles végétales; ouvrage en rapport avec les programmes officiels de l'Enseignement secondaire (1890).

Analyse descriptive des Rubus du Plateau Central (1891).

Supplément à la Flore d'Auvergne (1892).

Application de l'électricité atmosphérique à l'Agriculture (1892).

Note sur le dépôt diatomifère marin du Puy de Mur (1893), en collaboıtion avec M. A. Julien, professeur de géologie à l'Université de Clermont.

Les Diatomées d'Auvergne (1893). Ouvrage couronné par l'Institut de France (Académie des Sciences).

Influence de la lumière et de l'altitude sur la striation des valves des Diatomées (1894).

Nouvelles additions à la Flore d'Auvergne (1895).

Quelques mots sur la Truffe, au point de vue de sa nature, de sa culture et de son importance économique (1896).

Recherches sur les Diatomées des calcaires tertiaires de la Limagne et sur l'origine de ces terrains (1897).

Les Grimmia de la Flore d'Auvergne (1898).

Les Muscinées d'Auvergne (1899). Ouvrage couronné par l'Institut de France (Académie des Sciences).

La Flore d'Auvergne en 1901 (1902).

Un Garex nouveau pour la Flore française, en collaboration avec M. Jrnest Malinvaud, Secrétaire général de la Société botanique de France (1902).

Les Diatomées fossiles d suvergne (premier mémoire, 1902).

Les Diatomées fossiles d'Auvergne (second mémoire, 1903).

Pour paraitre prochainement: Flore miocène de Joursao (Cantal), en collaboration avec notre savant compatriote, M. Pierre Marty, le paléobotaniste cantalien bien connu, 


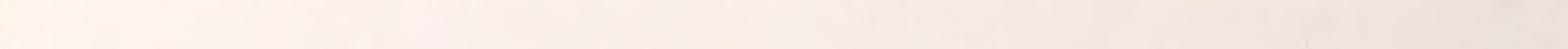

